\title{
On the minimal members of convex expectations with constraints
}

\author{
Rong-Lin Ji, Long Jiang* and De-Jian Tian
}

\author{
"Correspondence: \\ jianglong365@hotmail.com \\ Department of Mathematics, China \\ University of Mining and \\ Technology, Xuzhou, 221116 \\ P.R. China
}

\begin{abstract}
Let $S$ be a subset of all convex expectations containing all linear expectations. We prove that $\mathcal{E}$ is a minimal member of $S$ if and only if $\mathcal{E}$ is a linear expectation. Let $S$ be the set of all those convex expectations which are bigger than a concave expectation (resp. less than a convex expectation, sandwiched between a convex expectation and a concave expectation). We show that $\mathcal{E}$ is a minimal member of $S$ if and only if $\mathcal{E}$ is a linear expectation with the same constraints as above, respectively. This paper overcomes the deficiencies in the proofs of the main theorems in Huang and Jia (J. Math. Anal. Appl. 376:42-50, 2011) and generalizes the results in Huang and Jia (J. Math. Anal. Appl. 376:42-50, 2011).
\end{abstract}

Keywords: nonlinear expectation; sublinear expectation; convex expectation; minimal member

\section{Introduction}

It is well known that linear expectation is a powerful tool for dealing with stochastic phenomena. However, there are many uncertain phenomena which cannot be exactly measured by linear expectations. Scientists have found that the linearity causes the Allais paradox and Ellsberg paradox (see Allais (1953) and Ellsberg (1961)), and a lot of attention has been paid to nonlinear expectations and their applications. Peng [1] introduced the concept of $g$-expectations via nonlinear backward stochastic differential equations and showed that $g$-expectations are dynamically consistent (see also [2]). Coquet et al. [3] proposed an axiomatic approach to nonlinear expectations and introduced the notion of filtration-consistent nonlinear expectations. Chen et al. [4] investigated the connection between $g$-expectations and Choquet expectations, and Rosazza Gianin [5] and Jiang [6] investigated the relation between $g$-expectations and risk measures. Jia [7] studied the minimal members of sublinear expectations and their related properties, established the relationship between linear expectations and the minimal members of sublinear expectations, and obtained the so-called sandwich theorem for sublinear expectations and superlinear expectations. Furthermore, Huang and Jia [8] devoted their efforts to the study of the minimal members of convex expectations reaching conclusions similar to those in [7]. Unfortunately, there are some significant deficiencies in the course of the proofs of their main theorems in [8] (see Remark 3.1 and Remark 3.3).

The natural questions arise: do the results in [8] hold for convex expectations and what are the minimal members of convex expectations with some constraints?

(c) $2015 \mathrm{Ji}$ et al. This article is distributed under the terms of the Creative Commons Attribution 4.0 International License (http://creativecommons.org/licenses/by/4.0/), which permits unrestricted use, distribution, and reproduction in any medium, provided you give appropriate credit to the original author(s) and the source, provide a link to the Creative Commons license, and indicate if changes were made. 
The aim of this paper is to investigate the minimal members of nonlinear expectations in the framework of convex expectations with some constraints. We prove that, for a subset $S$ of all convex expectations containing all linear expectations, $\mathcal{E}$ is a minimal member of $S$ if and only if $\mathcal{E}$ is a linear expectation. Let $S$ be the set of all those convex expectations which are bigger than a concave expectation (resp. less than a convex expectation, sandwiched between a convex expectation and a concave expectation). We show that $\mathcal{E}$ is a minimal member of $S$ if and only if $\mathcal{E}$ is a linear expectation with the same constraints as above, respectively. In this article, our arguments overcome the deficiencies in the arguments in [8] and our results generalize the main results in [8].

The remainder of this paper is organized as follows: In Section 2, we introduce some notions, propositions, and lemmas which will be useful in this paper; in Section 3, we state and prove our main results.

\section{Preliminaries}

For the convenience of the readers, we recall some basic notions defined on a complete probability space $(\Omega, \mathcal{F}, P)$ as follows (see [8]).

A linear expectation $E[\cdot]$ is usually defined as a real functional $E: L^{1}(\Omega, \mathcal{F}, P) \longmapsto R$ with the following properties:

(i) $E[c]=c, \forall c \in R$;

(ii) $E[X] \geq E[Y]$ if $X \geq Y$ P-a.s; in addition, $P(X>Y)>0$ implies $E[X]>E[Y]$;

(iii) $E[\alpha X+\beta Y]=\alpha E[X]+\beta E[Y], \forall \alpha, \beta \in R$.

A nonlinear expectation $\mathcal{E}[\cdot]$ can also be defined as a real functional $\mathcal{E}: L^{1}(\Omega, \mathcal{F}, P) \longmapsto R$ satisfying the following conditions:

(A1) constant preserving: $\mathcal{E}[c]=c, \forall c \in R$

(A2) monotonicity: $\mathcal{E}[X] \geq \mathcal{E}[Y]$ if $X \geq Y$ P-a.s. Strict monotonicity: If $X \geq Y P$-a.s. and $P(X>Y)>0$, then $\mathcal{E}[X]>\mathcal{E}[Y]$.

A nonlinear expectation $\mathcal{E}[\cdot]$ is said to be sublinear if $\mathcal{E}$ satisfies:

(A3) (i) subadditivity: $\mathcal{E}[X+Y] \leq \mathcal{E}[X]+\mathcal{E}[Y]$;

(ii) positive homogeneity: $\mathcal{E}[\lambda X]=\lambda \mathcal{E}[X], \forall \lambda \geq 0$.

A nonlinear expectation $\mathcal{E}[\cdot]$ is said to be convex if $\mathcal{E}$ satisfies:

(A4) convexity: $\mathcal{E}[\alpha X+(1-\alpha) Y] \leq \alpha \mathcal{E}[X]+(1-\alpha) \mathcal{E}[Y], \forall \alpha \in[0,1]$.

A nonlinear expectation $\mathcal{E}[\cdot]$ is said to be superlinear if $\mathcal{E}$ satisfies:

(A5) (i) superadditivity: $\mathcal{E}[X+Y] \geq \mathcal{E}[X]+\mathcal{E}[Y]$;

(ii) positive homogeneity: $\mathcal{E}[\lambda X]=\lambda \mathcal{E}[X], \forall \lambda \geq 0$.

A nonlinear expectation $\mathcal{E}[\cdot]$ is called concave if $\mathcal{E}$ satisfies:

(A6) concavity: $\mathcal{E}[\alpha X+(1-\alpha) Y] \geq \alpha \mathcal{E}[X]+(1-\alpha) \mathcal{E}[Y], \forall \alpha \in[0,1]$.

Let $S$ be a subset of all nonlinear expectations. We say $\mathcal{E}_{0}$ a minimal member of $S$ if $\mathcal{E}_{0} \in S$ and $\mathcal{E}_{0}$ satisfy:

(A7) for any $\mathcal{E} \in S$, if $\mathcal{E}[X] \leq \mathcal{E}_{0}[X], \forall X \in L^{1}(\Omega, \mathcal{F}, P)$, then $\mathcal{E}=\mathcal{E}_{0}$.

Let $S$ be a subset of all nonlinear expectations. We call $\mathcal{E}_{0}$ a maximal member of $S$ if $\mathcal{E}_{0} \in S$ and $\mathcal{E}_{0}$ satisfy:

(A8) for any $\mathcal{E} \in S$, if $\mathcal{E}[X] \geq \mathcal{E}_{0}[X], \forall X \in L^{1}(\Omega, \mathcal{F}, P)$, then $\mathcal{E}=\mathcal{E}_{0}$.

For convenience, we denote the set of all linear expectations by $S^{1}$, the set of all sublinear expectations by $S^{\mathrm{sl}}$, the set of all convex expectations by $S^{\mathrm{cv}}$, the set of all superlinear expectations by $S^{\text {supl }}$, and the set of all concave expectations by $S^{\text {conca }}$. Clearly, $S^{1} \subset S^{\text {sl }} \subset S^{\text {cv }}, S^{1} \subset S^{\text {supl }} \subset S^{\text {conca }}$. For any two nonlinear expectations $\mathcal{E}_{1}$ and $\mathcal{E}_{2}, \mathcal{E}_{1} \geq \mathcal{E}_{2}$ means $\mathcal{E}_{1}[X] \geq \mathcal{E}_{2}[X]$ for any $X \in L^{1}(\Omega, \mathcal{F}, P)$. 
By the convexity (resp. concavity) and constant preserving condition, one can easily obtain Proposition 2.1, which shows that $S^{\mathrm{cv}}$ (resp. $S^{\mathrm{conca}}$ ) has minimal members (resp. maximal members). The proof is omitted.

Proposition 2.1 Let $E$ be a linear expectation. Then $E$ is a minimal member of $S^{\mathrm{cv}}$ and $a$ maximal member of $S^{\text {conca }}$.

The following lemmas come from Jia [7].

Lemma 2.1 Let $\mathcal{E} \in S^{\mathrm{sl}}$. Then the following statements are equivalent:

(i) $\mathcal{E}$ is a minimal member of $S^{\text {sl }}$;

(ii) $\mathcal{E}$ is a linear expectation.

Lemma 2.2 Let $\mathcal{E} \in S^{\mathrm{sl}}, U$ be a nonempty set of $L^{1}(\Omega, \mathcal{F}, P)$, and a functional $f: U \mapsto R$ be less than $\mathcal{E}$ on $U$. We define

$$
\mathcal{E}^{f}[X] \triangleq \inf _{Y \in U, \lambda \geq 0}\{\mathcal{E}[X+\lambda Y]-\lambda f(Y)\}, \quad X \in L^{1}(\Omega, \mathcal{F}, P)
$$

If $U$ is convex and $f$ is concave, then $\mathcal{E}^{f} \in S^{\mathrm{sl}}$.

\section{Main results}

Theorem 3.1 Let $S$ be a subset of $S^{\mathrm{cv}}$ satisfying $S^{\mathrm{l}} \subseteq S$. Let $\mathcal{E}_{0} \in S$. Then the following two statements are equivalent:

(i) $\mathcal{E}_{0}$ is a minimal member of $S$;

(ii) $\mathcal{E}_{0}$ is a linear expectation.

Proof Since $S^{1} \subseteq S$, Proposition 2.1 shows that $S$ has at least a minimal member and (ii) $\Rightarrow$ (i) holds.

(i) $\Rightarrow$ (ii) Since $\mathcal{E}_{0}$ is a convex expectation, we define

$$
\mathcal{E}_{0}^{*}[X] \triangleq \lim _{\lambda \rightarrow+\infty} \lambda \mathcal{E}_{0}\left[\frac{X}{\lambda}\right], \quad \forall X \in L^{1}(\Omega, \mathcal{F}, P)
$$

Then we assert that $\mathcal{E}_{0}^{*}$ is well defined and

$$
\mathcal{E}_{0}^{*} \in S^{\mathrm{sl}} \subset S^{\mathrm{cv}} \text {. }
$$

First, let us prove that $\mathcal{E}_{0}^{*}$ is real-valued. In fact, setting $\lambda_{1} \geq \lambda_{2}>0$, then $k \triangleq \frac{\lambda_{1}}{\lambda_{2}}$ and $\frac{1}{k} \in(0,1]$. Noticing that $\mathcal{E}_{0}$ is convex, for each $X \in L^{1}(\Omega, \mathcal{F}, P)$ we have

$$
\begin{aligned}
\lambda_{1} \mathcal{E}_{0}\left[\frac{X}{\lambda_{1}}\right] & =k \lambda_{2} \mathcal{E}_{0}\left[\frac{X}{k \lambda_{2}}\right] \\
& \leq k \lambda_{2} \frac{1}{k} \mathcal{E}_{0}\left[\frac{X}{\lambda_{2}}\right] \\
& =\lambda_{2} \mathcal{E}_{0}\left[\frac{X}{\lambda_{2}}\right]
\end{aligned}
$$


Thus, $\lambda \mathcal{E}_{0}\left[\frac{X}{\lambda}\right]$ is a decreasing function of $\lambda$ on $(0,+\infty)$ for each $X \in L^{1}(\Omega, \mathcal{F}, P)$. Taking $\lambda=1$ in (1) yields

$$
\mathcal{E}_{0}^{*}[X] \leq \mathcal{E}_{0}[X], \quad \forall X \in L^{1}(\Omega, \mathcal{F}, P)
$$

Furthermore, it is easy to see that $-\lambda \mathcal{E}_{0}\left[\frac{-X}{\lambda}\right]$ is an increasing function of $\lambda$ on $(0,+\infty)$ for each $X \in L^{1}(\Omega, \mathcal{F}, P)$. Since $\mathcal{E}_{0}$ satisfies the constant preserving and convexity conditions, we have

$$
\begin{aligned}
\mathcal{E}_{0}^{*}[X] & =\lim _{\lambda \rightarrow+\infty} \lambda \mathcal{E}_{0}\left[\frac{X}{\lambda}\right] \\
& \geq \lim _{\lambda \rightarrow+\infty}-\lambda \mathcal{E}_{0}\left[\frac{-X}{\lambda}\right] \\
& \geq-\mathcal{E}_{0}[-X] \\
& >-\infty .
\end{aligned}
$$

Hence $\mathcal{E}_{0}^{*}[X]$ is real-valued on $L^{1}(\Omega, \mathcal{F}, P)$.

Second, let us prove $\mathcal{E}_{0}^{*} \in S^{\text {sl }} \subset S^{\text {cv }}$. It is obvious that

$$
\begin{aligned}
& \mathcal{E}_{0}^{*}[c]=c, \quad \forall c \in R, \\
& \mathcal{E}_{0}^{*}[X] \geq \mathcal{E}_{0}^{*}[Y], \quad \text { if } X \geq Y, P \text {-a.s. }
\end{aligned}
$$

We show that $\mathcal{E}_{0}^{*}$ satisfies the convexity condition. Indeed, since $\mathcal{E}_{0}^{*}$ is real-valued and $\mathcal{E}_{0}$ is convex, for all $\alpha \in[0,1], X, Y \in L^{1}(\Omega, \mathcal{F}, P)$, we deduce that

$$
\begin{aligned}
\mathcal{E}_{0}^{*}[\alpha X+(1-\alpha) Y] & =\lim _{\lambda \rightarrow+\infty} \lambda \mathcal{E}_{0}\left[\alpha \frac{X}{\lambda}+(1-\alpha) \frac{Y}{\lambda}\right] \\
& \leq \lim _{\lambda \rightarrow+\infty} \lambda\left(\alpha \mathcal{E}_{0}\left[\frac{X}{\lambda}\right]+(1-\alpha) \mathcal{E}_{0}\left[\frac{Y}{\lambda}\right]\right) \\
& =\lim _{\lambda \rightarrow+\infty} \alpha \lambda \mathcal{E}_{0}\left[\frac{X}{\lambda}\right]+\lim _{\lambda \rightarrow+\infty}(1-\alpha) \lambda \mathcal{E}_{0}\left[\frac{Y}{\lambda}\right] \\
& =\alpha \mathcal{E}_{0}^{*}[X]+(1-\alpha) \mathcal{E}_{0}^{*}[Y] .
\end{aligned}
$$

Now we prove that

$$
\mathcal{E}_{0}^{*}[\beta X]=\beta \mathcal{E}_{0}^{*}[X], \quad \forall \beta \geq 0, X \in L^{1}(\Omega, \mathcal{F}, P) .
$$

Since $\mathcal{E}_{0}^{*}$ is real-valued and $\mathcal{E}_{0}^{*}[0]=0, \beta=0$ implies

$$
\mathcal{E}_{0}^{*}[\beta X]=0=\beta \mathcal{E}_{0}^{*}[X]
$$

Let $\beta>0$. We conclude that

$$
\begin{aligned}
\mathcal{E}_{0}^{*}[\beta X] & =\lim _{\lambda \rightarrow+\infty} \lambda \mathcal{E}_{0}\left[\beta \frac{X}{\lambda}\right] \\
& =\lim _{\beta \lambda \rightarrow+\infty} \beta \lambda \mathcal{E}_{0}\left[\frac{X}{\lambda}\right]
\end{aligned}
$$




$$
\begin{aligned}
& =\beta \lim _{\lambda \rightarrow+\infty} \lambda \mathcal{E}_{0}\left[\frac{X}{\lambda}\right] \\
& =\beta \mathcal{E}_{0}^{*}[X] .
\end{aligned}
$$

For any $X, Y \in L^{1}(\Omega, \mathcal{F}, P),(6)$ and (7) imply that

$$
\mathcal{E}_{0}^{*}[X+Y] \leq \mathcal{E}_{0}^{*}[X]+\mathcal{E}_{0}^{*}[Y]
$$

Let $X \geq Y P$-a.s. and $P(X>Y)>0$. In view of (3), (8), and the strict monotonicity of $\mathcal{E}_{0}$, we deduce that

$$
\begin{aligned}
\mathcal{E}_{0}^{*}[Y]-\mathcal{E}_{0}^{*}[X] & \leq \mathcal{E}_{0}^{*}[Y-X] \\
& \leq \mathcal{E}_{0}[Y-X] \\
& <\mathcal{E}_{0}[0] \\
& =0 .
\end{aligned}
$$

Since $\mathcal{E}_{0}^{*}$ is real-valued, combining (4), (5), (7), (8) with (9), we get $\mathcal{E}_{0}^{*} \in S^{\text {sl }} \subset S^{\text {cv }}$.

Since $\mathcal{E}_{0}$ is a minimal member of $S$, applying Lemma 2.1, we know that there exists a linear expectation $E \in S^{1}$ satisfying $E \leq \mathcal{E}_{0}^{*} \leq \mathcal{E}_{0}$. Noticing that $E \in S^{\mathrm{l}} \subseteq S$ and $\mathcal{E}_{0}$ is a minimal member of $S$, we have $\mathcal{E}_{0}=E \in S^{1}$.

Remark 3.1 In [8], Huang and Jia applied Lemma 2.11 to prove their main theorem (Theorem 2.12). Unfortunately, they cannot assert that the minimal members of convex expectations must be sublinear in that lemma (see line 13 of p.48).

Remark 3.2 Let $\mathcal{E}_{1}$ be a convex expectation and $\mathcal{E}_{2}$ be a concave expectation with $\mathcal{E}_{1} \geq \mathcal{E}_{2}$. Let $\mathcal{E}_{1}^{*}$ and $\mathcal{E}_{2}^{*}$ be defined as in (1). Then it is easy to verify that $\mathcal{E}_{1}^{*}$ is a sublinear expectation and $\mathcal{E}_{2}^{*}$ is a superlinear expectation with $\mathcal{E}_{1} \geq \mathcal{E}_{1}^{*} \geq \mathcal{E}_{2}^{*} \geq \mathcal{E}_{2}$.

With the help of Theorem 3.1, we immediately have the following corollary.

Corollary 3.1 Let $S$ be the set of all convex expectations with comonotonic additivity. Then $\mathcal{E}_{0}$ is a minimal member of $S$ if and only if $\mathcal{E}_{0}$ is a linear expectation.

In the following, we will discuss the minimal members of three kinds of subsets of $S^{\mathrm{cv}}$. In Theorem 3.2, we investigate the minimal members of the set of all those convex expectations which are smaller than a given convex expectation; in Theorem 3.3, we study the minimal members of the set of all those convex expectations which are bigger than a given concave expectation; and we consider the minimal members of the set of all those convex expectations which are sandwiched between a convex expectation and a concave expectation in Theorem 3.4.

Theorem 3.2 Let $\mathcal{E}_{0} \in S^{\mathrm{cv}}$. We set

$$
S^{\mathrm{cv}}\left(\mathcal{E}_{0}\right):=\left\{\mathcal{E}: \mathcal{E} \leq \mathcal{E}_{0}, \mathcal{E} \in S^{\mathrm{cv}}\right\}
$$

Then $\mathcal{E}$ is a minimal member of $S^{\mathrm{cv}}\left(\mathcal{E}_{0}\right)$ if and only if $\mathcal{E} \in S^{1} \cap S^{\mathrm{cv}}\left(\mathcal{E}_{0}\right)$. 
Proof Since $\mathcal{E}_{0} \in S^{\mathrm{cv}}$, applying Theorem 3.1 there exists a linear expectation $E_{1} \in S^{1}$ satisfying $E_{1} \leq \mathcal{E}_{0}$. Thus $E_{1} \in S^{\mathrm{l}} \cap S^{\mathrm{cv}}\left(\mathcal{E}_{0}\right)$. For each $E \in S^{\mathrm{l}} \cap S^{\mathrm{cv}}\left(\mathcal{E}_{0}\right)$, Theorem 3.1 implies that $E$ is a minimal member of $S^{\mathrm{cv}}\left(\mathcal{E}_{0}\right)$.

We now show that, for each minimal member $\mathcal{E}$ of $S^{\mathrm{cv}}\left(\mathcal{E}_{0}\right), \mathcal{E} \in S^{\mathrm{l}} \cap S^{\mathrm{cv}}\left(\mathcal{E}_{0}\right)$. In fact, noticing that $\mathcal{E} \in S^{\mathrm{cv}}\left(\mathcal{E}_{0}\right)$, with the help of Theorem 3.1, we know that there exists a linear expectation $E_{2} \in S^{1}$ with $E_{2} \leq \mathcal{E}$. Clearly, $E_{2} \leq \mathcal{E}_{0}$ and $E_{2} \in S^{1} \cap S^{\mathrm{cv}}\left(\mathcal{E}_{0}\right)$, thus $\mathcal{E}=E_{2} \in$ $S^{\mathrm{l}} \cap S^{\mathrm{cv}}\left(\mathcal{E}_{0}\right)$.

Analogously to the argument for the minimal members of convex expectations, we have the following result on concave expectations.

Proposition 3.1 Let $S$ be a subset of $S^{\text {conca }}$ satisfying $S^{1} \subseteq S$. Let $\mathcal{E}_{0} \in S$. Then the following two statements are equivalent:

(i) $\mathcal{E}_{0}$ is a maximal member of $S$;

(ii) $\mathcal{E}_{0}$ is a linear expectation.

Theorem 3.3 Let $\mathcal{E}_{0}$ be a concave expectation. Let

$$
S^{\mathrm{cv}}\left(\mathcal{E}_{0}\right):=\left\{\mathcal{E}: \mathcal{E} \geq \mathcal{E}_{0}, \mathcal{E} \in S^{\mathrm{cv}}\right\}
$$

Then $\mathcal{E}$ is a minimal member of $S^{\mathrm{cv}}\left(\mathcal{E}_{0}\right)$ if and only if $\mathcal{E} \in S^{\mathrm{l}} \cap S^{\mathrm{cv}}\left(\mathcal{E}_{0}\right)$.

Proof By Proposition 3.1, for a given concave expectation $\mathcal{E}_{0}$, there exists a linear expectation $E_{1} \in S^{\mathrm{l}}$ such that $E_{1} \geq \mathcal{E}_{0}$. Thus $E_{1} \in S^{\mathrm{l}} \cap S^{\mathrm{cv}}\left(\mathcal{E}_{0}\right)$. For each $E \in S^{\mathrm{l}} \cap S^{\mathrm{cv}}\left(\mathcal{E}_{0}\right)$, by Theorem 3.1 we know that $E$ is a minimal member of $S^{\mathrm{cv}}\left(\mathcal{E}_{0}\right)$.

Now we prove that, for each minimal member $\mathcal{E}$ of $S^{\mathrm{cv}}\left(\mathcal{E}_{0}\right), \mathcal{E} \in S^{\mathrm{l}} \cap S^{\mathrm{cv}}\left(\mathcal{E}_{0}\right)$. Clearly, $\mathcal{E} \in S^{\text {cv }}$ and $\mathcal{E} \geq \mathcal{E}_{0}$. By Remark 3.2, we have $\mathcal{E}^{*} \in S^{\text {sl }}, \mathcal{E}_{0}^{*} \in S^{\text {supl }}$, and $\mathcal{E} \geq \mathcal{E}^{*} \geq \mathcal{E}_{0}^{*} \geq \mathcal{E}_{0}$. If there exists a linear expectation $E$ satisfying $\mathcal{E}^{*} \geq E \geq \mathcal{E}_{0}^{*}$, then we obtain $E \in S^{\mathrm{l}} \cap S^{\mathrm{cv}}\left(\mathcal{E}_{0}\right)$, and with the assumption that $\mathcal{E}$ is a minimal member of $S^{\mathrm{cv}}\left(\mathcal{E}_{0}\right)$, we immediately deduce that $\mathcal{E}=E \in S^{\mathrm{l}} \cap S^{\mathrm{cv}}\left(\mathcal{E}_{0}\right)$. So we only need to prove that there exists a linear expectation $E$ satisfying $\mathcal{E}^{*} \geq E \geq \mathcal{E}_{0}^{*}$.

For simplicity, we set

$$
S^{\mathrm{sl}}\left(\mathcal{E}_{0}^{*}\right):=\left\{\overline{\mathcal{E}}: \overline{\mathcal{E}} \geq \mathcal{E}_{0}^{*}, \overline{\mathcal{E}} \in S^{\mathrm{sl}}\right\} .
$$

In what follows, we show that $S^{\text {sl }}\left(\mathcal{E}_{0}^{*}\right)$ has at least a minimal member, and $\overline{\mathcal{E}}$ is a minimal member of $S^{\text {sl }}\left(\mathcal{E}_{0}^{*}\right)$ if and only if $\overline{\mathcal{E}} \in S^{1} \cap S^{\mathrm{sl}}\left(\mathcal{E}_{0}^{*}\right)$.

Thanks to Proposition 3.1, there exists a linear expectation $E_{2} \in S^{1}$ such that $E_{2} \geq \mathcal{E}_{0}^{*}$. Thus $E_{2} \in S^{\mathrm{l}} \cap S^{\mathrm{sl}}\left(\mathcal{E}_{0}^{*}\right)$. With the help of Lemma 2.1, we know that for each $E \in S^{\mathrm{l}} \cap S^{\mathrm{sl}}\left(\mathcal{E}_{0}^{*}\right)$, $E$ is a minimal member of $S^{\text {sl }}\left(\mathcal{E}_{0}^{*}\right)$.

We now prove that, for each minimal member $\overline{\mathcal{E}}$ of $S^{\text {sl }}\left(\mathcal{E}_{0}^{*}\right), \overline{\mathcal{E}} \in S^{\mathrm{l}} \cap S^{\mathrm{sl}}\left(\mathcal{E}_{0}^{*}\right)$.

Step 1: We show that $\overline{\mathcal{E}}[X+Y] \geq \overline{\mathcal{E}}[X]+\mathcal{E}_{0}^{*}[Y], \forall X, Y \in L^{1}(\Omega, \mathcal{F}, P)$.

For each fixed $Y \in L^{1}(\Omega, \mathcal{F}, P)$, we define

$$
\overline{\mathcal{E}}^{\mathcal{E}_{0}^{*}}[X] \triangleq \inf _{\lambda \geq 0}\left\{\overline{\mathcal{E}}[X+\lambda Y]-\lambda \mathcal{E}_{0}^{*}[Y]\right\}, \quad X \in L^{1}(\Omega, \mathcal{F}, P)
$$


By Lemma 2.2, we have $\overline{\mathcal{E}}^{\mathcal{E}_{0}^{*}} \in S^{\text {sl }}$ and $\overline{\mathcal{E}}^{\mathcal{E}_{0}^{*}} \leq \overline{\mathcal{E}}$. In view of the fact

$$
\overline{\mathcal{E}}[X+\lambda Y]-\lambda \mathcal{E}_{0}^{*}[Y] \geq \mathcal{E}_{0}^{*}[X+\lambda Y]-\lambda \mathcal{E}_{0}^{*}[Y] \geq \mathcal{E}_{0}^{*}[X]
$$

we conclude that $\overline{\mathcal{E}}^{*} \in S^{\text {sl }}\left(\mathcal{E}_{0}^{*}\right)$. Hence $\overline{\mathcal{E}}=\overline{\mathcal{E}}^{*}$. Taking $\lambda=1$ in (10) yields

$$
\overline{\mathcal{E}}[X+Y] \geq \overline{\mathcal{E}}[X]+\mathcal{E}_{0}^{*}[Y], \quad \forall X, Y \in L^{1}(\Omega, \mathcal{F}, P) .
$$

Step 2: We prove that $\overline{\mathcal{E}}[X+Y] \geq \overline{\mathcal{E}}[X]+\overline{\mathcal{E}}[Y], \forall X, Y \in L^{1}(\Omega, \mathcal{F}, P)$.

For every fixed $Y \in L^{1}(\Omega, \mathcal{F}, P)$, we define

$$
\overline{\mathcal{E}}^{\overline{\mathcal{E}}}[X] \triangleq \inf _{\lambda \geq 0}\{\overline{\mathcal{E}}[X+\lambda Y]-\lambda \overline{\mathcal{E}}[Y]\}, \quad X \in L^{1}(\Omega, \mathcal{F}, P) .
$$

Applying Lemma 2.2, we have $\overline{\mathcal{E}}^{\overline{\mathcal{E}}} \in S^{\mathrm{sl}}$ and $\overline{\mathcal{E}}^{\overline{\mathcal{E}}} \leq \overline{\mathcal{E}}$. Noticing that

$$
\overline{\mathcal{E}}[X+Y] \geq \overline{\mathcal{E}}[X]+\mathcal{E}_{0}^{*}[Y], \quad \forall X, Y \in L^{1}(\Omega, \mathcal{F}, P),
$$

we obtain $\overline{\mathcal{E}}[X+\lambda Y]-\lambda \overline{\mathcal{E}}[Y] \geq \mathcal{E}_{0}^{*}[X]+\overline{\mathcal{E}}[\lambda Y]-\lambda \overline{\mathcal{E}}[Y]=\mathcal{E}_{0}^{*}[X]$. Thus $\overline{\mathcal{E}}^{\overline{\mathcal{E}}} \in S^{\text {sl }}\left(\mathcal{E}_{0}^{*}\right)$ and $\overline{\mathcal{E}}=\overline{\mathcal{E}}^{\overline{\mathcal{E}}}$. Let $\lambda=1$. It follows from (12) that

$$
\overline{\mathcal{E}}[X+Y] \geq \overline{\mathcal{E}}[X]+\overline{\mathcal{E}}[Y], \quad \forall X, Y \in L^{1}(\Omega, \mathcal{F}, P)
$$

Step 3: We prove that $\overline{\mathcal{E}} \in S^{1} \cap S^{\text {sl }}\left(\mathcal{E}_{0}^{*}\right)$.

Thanks to $\overline{\mathcal{E}}[X+Y] \geq \overline{\mathcal{E}}[X]+\overline{\mathcal{E}}[Y], \forall X, Y \in L^{1}(\Omega, \mathcal{F}, P)$, we have

$$
\overline{\mathcal{E}}[X+Y]=\overline{\mathcal{E}}[X]+\overline{\mathcal{E}}[Y], \quad \forall X, Y \in L^{1}(\Omega, \mathcal{F}, P) .
$$

Since $\overline{\mathcal{E}}$ satisfies constant preserving, we obtain $\overline{\mathcal{E}}[X]=-\overline{\mathcal{E}}[-X]$, which means $\overline{\mathcal{E}}[\lambda X]=$ $\lambda \overline{\mathcal{E}}[X], \forall \lambda \in R, X \in L^{1}(\Omega, \mathcal{F}, P)$. Thus $\overline{\mathcal{E}} \in S^{\mathrm{l}}$. Noticing that $\overline{\mathcal{E}} \geq \mathcal{E}_{0}^{*}$, we have $\overline{\mathcal{E}} \in S^{\mathrm{l}} \cap S^{\mathrm{sl}}\left(\mathcal{E}_{0}^{*}\right)$.

Since $\mathcal{E}^{*} \in S^{\mathrm{sl}}\left(\mathcal{E}_{0}^{*}\right)$, there naturally exists a linear expectation $E \in S^{\mathrm{l}} \cap S^{\mathrm{sl}}\left(\mathcal{E}_{0}^{*}\right)$ such that $\mathcal{E}^{*} \geq E \geq \mathcal{E}_{0}^{*}$.

Remark 3.3 In [8], Huang and Jia applied the basic Lemma 2.3 and the famous Zorn lemma to show the existence of minimal members in the course of the proof of their other main theorem (Theorem 3.1) (see line 33 of p.48). But the strict monotonicity condition of the operator in Lemma 2.3 has not yet been proved (see line 16 of p.44). Moreover, in the further proof of their Theorem 3.1, the question whether the operator belongs to the set remains to be answered (see line 22 of p.49).

From Theorem 3.3 one immediately deduces the following corollary, which is usually called a sandwich theorem. In a sense, such a result can be regarded as the Hahn-Banach theorem of nonlinear expectations (see [9]).

Corollary 3.2 Let $\mathcal{E}_{1}$ be a convex expectation and $\mathcal{E}_{2}$ be a concave expectation. If $\mathcal{E}_{1} \geq \mathcal{E}_{2}$, then there exists a linear expectation $E$ such that $\mathcal{E}_{1} \geq E \geq \mathcal{E}_{2}$ (see Theorem 3.1 in [8]). 
Theorem 3.4 Let $\mathcal{E}_{0}$ be a convex expectation, $\mathcal{E}_{1}$ be a concave expectation with $\mathcal{E}_{1} \leq \mathcal{E}_{0}$.

We set

$$
S^{\mathrm{cv}}\left(\mathcal{E}_{0}, \mathcal{E}_{1}\right):=\left\{\mathcal{E}: \mathcal{E}_{1} \leq \mathcal{E} \leq \mathcal{E}_{0}, \mathcal{E} \in S^{\mathrm{cv}}\right\}
$$

Then $\mathcal{E}$ is a minimal member of $S^{\mathrm{cv}}\left(\mathcal{E}_{0}, \mathcal{E}_{1}\right)$ if and only if $\mathcal{E} \in S^{\mathrm{l}} \cap S^{\mathrm{cv}}\left(\mathcal{E}_{0}, \mathcal{E}_{1}\right)$.

Proof Corollary 3.2 implies that there exists a linear expectation $E_{1}$ satisfying $\mathcal{E}_{1} \leq E_{1} \leq \mathcal{E}_{0}$, thus $E_{1} \in S^{\mathrm{l}} \cap S^{\mathrm{cv}}\left(\mathcal{E}_{0}, \mathcal{E}_{1}\right)$. For each $E \in S^{\mathrm{l}} \cap S^{\mathrm{cv}}\left(\mathcal{E}_{0}, \mathcal{E}_{1}\right)$, by Theorem 3.1 we know that $E$ is a minimal member of $S^{\mathrm{cv}}\left(\mathcal{E}_{0}, \mathcal{E}_{1}\right)$. For any minimal member $\mathcal{E}$ of $S^{\mathrm{cv}}\left(\mathcal{E}_{0}, \mathcal{E}_{1}\right)$, clearly, $\mathcal{E}_{1} \leq \mathcal{E} \leq$ $\mathcal{E}_{0}$ and $\mathcal{E} \in S^{\mathrm{cv}}$. Applying Corollary 3.2 again, there exists a linear expectation $E_{2}$ satisfying $\mathcal{E}_{1} \leq E_{2} \leq \mathcal{E} \leq \mathcal{E}_{0}$, hence $\mathcal{E}=E_{2} \in S^{\mathrm{l}} \cap S^{\mathrm{cv}}\left(\mathcal{E}_{0}, \mathcal{E}_{1}\right)$.

\section{Competing interests}

The authors declare that they have no competing interests.

\section{Authors' contributions}

All authors read and approved the final manuscript.

\section{Acknowledgements}

The authors are thankful to the anonymous referees for their valuable comments and suggestions, which helped to improve the presentation of this paper. The work was supported by the National Natural Science Foundation of China (No. 11371362), the Fundamental Research Funds for the Central Universities (No. 2012LWB48), and the Research Innovation Program for College Graduates of Jiangsu Province (No. KYZZ_0373).

Received: 28 October 2014 Accepted: 28 May 2015 Published online: 12 June 2015

\section{References}

1. Peng, SG: BSDE and related g-expectation. In: Karoui, EN, Mazliak, L (eds.) Backward Stochastic Differential Equations. Pitman Res. Notes Math. Ser., vol. 364, pp. 141-159. Longman, Harlow (1997)

2. Peng, SG: Nonlinear Expectations, Nonlinear Evaluations and Risk Measures. Springer, Berlin (2004)

3. Coquet, F, Hu, Y, Mémin, J, Peng, SG: Filtration-consistent nonlinear expectations and related $g$-expectations. Probab. Theory Relat. Fields 123, 1-27 (2002)

4. Chen, ZJ, Chen, T, Davison, M: Choquet expectation and Peng's g-expectation. Ann. Probab. 33(3), 1179-1199 (2005)

5. Rosazza Gianin, E: Risk measures via g-expectations. Insur. Math. Econ. 39, 19-34 (2006)

6. Jiang, L: Convexity, translation invariance and subadditivity for $g$-expectations and related risk measures. Ann. Appl. Probab. 18, 245-258 (2008)

7. Jia, GY: The minimal sublinear expectations and their related properties. Sci. China Math. 39, 79-87 (2009)

8. Huang, J, Jia, GY: On the minimal members of convex expectations. J. Math. Anal. Appl. 376, 42-50 (2011)

9. Yosida, K: Functional Analysis. Springer, Berlin (1980)

\section{Submit your manuscript to a SpringerOpen ${ }^{\circ}$ journal and benefit from:}

- Convenient online submission

Rigorous peer review

- Immediate publication on acceptance

- Open access: articles freely available online

- High visibility within the field

- Retaining the copyright to your article 